\title{
EIGENFUNCTION EXPANSION METHOD FOR MULTIPLE SOLUTIONS OF FOURTH-ORDER ORDINARY DIFFERENTIAL EQUATIONS WITH CUBIC POLYNOMIAL NONLINEARITY*
}

\author{
ABDRHAMAN MAHMOUD ${ }^{\dagger}$, BO $\mathrm{YU}^{\dagger}$, AND XUPING ZHANG ${ }^{\dagger}$
}

\begin{abstract}
Multiple solutions of fourth-order ordinary differential equations (ODEs) with cubic polynomial nonlinearity are presented in this paper. The ODEs are discretized by the eigenfunction expansion method. Discretization error estimates are derived. We construct an efficient polynomial homotopy to find all solutions for the system of polynomial equations on the coarse level by recursion. Two kinds of filters are suggested for removing possible spurious solutions of the discretized system of polynomial equations. Numerical experiments are included to verify the error estimates and efficiency of the proposed homotopy.
\end{abstract}

Key words. fourth-order ODEs, eigenfunction expansion method, system of polynomial equations, multiple solutions, homotopy continuation method

AMS subject classifications. 34L10, 34B15, 65H04

1. Introduction. In this paper, our aim is to find numerically multiple solutions for the following nonlinear fourth-order differential equation

$$
y^{(4)}+\alpha y^{\prime \prime}+\beta y=f(y)+g(x), \quad x \in \Omega \equiv(0,1)
$$

with the boundary conditions

$$
y(0)=y(1)=y^{\prime \prime}(0)=y^{\prime \prime}(1)=0,
$$

where $\alpha$ and $\beta$ represent some real constants, $f$ is a polynomial of $y$ and $g \in L^{2}(\Omega)$ is a given function.

The boundary value problem (BVP) (1.1) usually describes the equilibrium state of the deformation of an elastic beam with supported ends. This problem has been widely addressed by researchers using various types of equations and boundary conditions $[2,4,10]$. The relevant applications of fourth-order linear and nonlinear ODE models have gained considerable attention in areas such as biology, physics, and engineering [6, 13, 29]. Nonlinear science entails a difficult aspect in the application of nonlinear mathematical problems.

The difficulties of multiple solution problems involve theoretical issues, such as distribution and structure of the solution set. It also involves algorithmic issues, such as discretization methods for the differential equations and algebraic methods for the discretized equations. Usually, it is quite difficult to obtain closed form solutions for BVPs, especially for nonlinear differential equations. Only in certain scenarios, analytic solutions can be anticipated. Hence, numerical solutions for these problems become a choice. The solutions for problem (1.1) are mostly dependent on the nonlinearity of $f$. Therefore, the number of solutions and their existence are related to the growth conditions of nonlinearity; see [5, 14, 18, 21].

Many researchers deal with determining the numerical and analytic solutions of fourthorder ordinary differential equations to both linear and nonlinear equations with initial and/or boundary conditions. Numerical solutions are computed by the Finite Element Method [11],

* Received April 26, 2017. Accepted January 10, 2018. Published online on April 5, 2018. Recommended by F. Marcellan.

${ }^{\dagger}$ School of Mathematical Sciences, Dalian University of Technology, Liaoning 116025, Dalian, China (Abdelrhaman013eyahoo.com, yuboedlut.edu.cn, zhangxp@dlut.edu.cn).

${ }^{\ddagger}$ Department of Mathematics, Faculty of Sciences and Technology, Omdurman Islamic University, Omdurman, Sudan. 
the Finite Difference Method [26], and the Sinc-Galerkin Method [23], while analytic solutions are obtained by the Homotopy Perturbation Method [19], the Homotopy Analysis Method [17], the Lower and Upper Solution Method [22], and the Modified Decomposition Method [28]. As far as we know from the literature, there are only a few works on finding multiple solutions of differential equations and even fewer for partial differential equations.

The Search-Extension Method (SEM) computes all possible solutions of semilinear elliptic equations, which was suggested by Chen and Xie [7]. They also studied important aspects of SEM for their significance by considering square, unit circle, and L-shape domains. In SEM, the eigenfunction expansion method (EEM) is used as discretization method, where the eigenfunctions are those of the Laplacian $-\Delta$. The main aspect of SEM consists of two steps: First, all solutions for the EEM system in a small subspace, based on the cuboid algorithm, are searched. Second, the approximate solutions obtained from the first step are used as initial points in the numerical extension method (homotopy method) for further computation of multiple solutions associated with the EEM system in a larger subspace.

Allgower et al. [1] established numerical continuation methods for the finite difference discretization of nonlinear two-point boundary value problems. They performed a homotopy deformation on successively refined discretization systems in order to obtain solutions on the finer level. Some filters are used to remove spurious solutions that lead to an efficient homotopy. These filters depend on the information of the original problem and no general rule seems to be available.

By using the eigenfunction expansion method, the multiple solutions of semilinear elliptic equations with polynomial nonlinearity have been investigated by Zhang et al. [31]. The solutions for the discretized problem on a coarse level were computed and used as initial guesses to find solutions of the discretized problem on a finer level. For the resulting system of polynomial equations, they suggested the extension homotopy method to find efficiently all solutions. They proposed a filter strategy to remove possible spurious solutions that depends on the error estimates of the eigenfunction expansion discretization. Due to the high computational cost, the dimension of the coarse level discretization is usually low. The computed solutions can then be refined by the finite element method or the finite difference method.

In this paper, we find multiple solutions of the nonlinear fourth-order differential equation by the Eigenfunction Expansion Method. We formulate Algorithm 1 for the given system of polynomial equations, which can be solved by software packages from the numerical algebraic geometry community such as PHC, HOM4PS-2.0, PHoM, and Bertini [3, 12, 15, 16, 27]. The solution set of the discretization system may have spurious solutions, which are not close to the original solutions. Therefore, we apply the central difference scheme as a filter to remove spurious solutions appearing in the solution set of the discretized system. We also apply Newton's method as a filter to remove spurious solutions.

This paper is organized as follows: In Section 2, we discretize the fourth-order differential equation with polynomial nonlinearity by using the eigenfunction expansion method. In Section 3, we constructed a polynomial homotopy to find all solutions for the resulting system of polynomial equations on the coarse level. Section 4 contains the derivation of error estimates for the discretization method. In Section 5, we provide filters for removing spurious solutions. In Section 6, the details of our computation and numerical examples are presented to confirm the accuracy of the eigenfunction expansion method and the efficiency of the homotopy method. Conclusions are drawn in Section 7.

2. Discretization by the eigenfunction expansion method. The weak formulation of (1.1) with homogeneous boundary conditions is defined as follows: find $y \in H^{2}(\Omega) \cap H_{0}^{1}(\Omega)$, 
such that

$$
\begin{array}{r}
A(y, \phi)=\int_{0}^{1}\left(y^{\prime \prime} \phi^{\prime \prime}-\alpha y^{\prime} \phi^{\prime}+\beta y \phi\right) d x-\int_{0}^{1} f(y) \phi d x-\int_{0}^{1} g(x) \phi d x=0, \\
\forall \phi \in H^{2}(\Omega) \cap H_{0}^{1}(\Omega),
\end{array}
$$

where $H^{2}(\Omega) \cap H_{0}^{1}(\Omega)$ is a Sobolev space with the first boundary condition $y(0)=y(1)=0$.

The general idea of the method is to choose the set of eigenfunctions $\left\{\phi_{i}\right\}_{i=1}^{\infty}$ corresponding to the Sturm-Liouville operator with boundary conditions that satisfies

$$
\begin{aligned}
H\left[\phi_{i}\right] & =\lambda_{i} \phi_{i} \\
R_{k}\left[\phi_{i}\right] & =0, \quad k=1,2,3, \ldots, n,
\end{aligned}
$$

where $H[\cdot]$ is a linear differential operator related to $(1.1), R_{k}[\cdot]$ are linear boundary conditions and $\lambda_{i}$ are eigenvalues of the system $\left(H,\left[R_{k}\right]\right)$. Specifically, the eigenpairs $\left\{\lambda_{i}, \phi_{i}\right\}$ of the fourth-order differential operator with boundary conditions corresponding to (1.1) are as follows,

$$
\left\{\begin{array}{l}
\phi^{\prime \prime \prime \prime}=\lambda \phi \\
\phi(0)=\phi(1)=\phi^{\prime \prime}(0)=\phi^{\prime \prime}(1)=0 .
\end{array}\right.
$$

Thus, we have solutions $\phi_{i}(x)=C \cdot \sin (i \pi x)$, where $C$ is an arbitrary constant.

The set of eigenfunctions $\left\{\phi_{i}\right\}_{i=1}^{\infty}$ is a normalized orthogonal basis of the Sobolev space $V(\Omega):=H^{2}(\Omega) \cap H_{0}^{1}(\Omega)$. Indeed, the orthogonality condition requires that $\left(\phi_{i}, \phi_{j}\right)=\delta_{i j}$, where $\delta_{i j}$ is the Kronecker delta. Since the $\phi_{i}$ are normalized, the eigenpairs are as follows

$$
\phi_{i}(x)=\sqrt{2} \sin (i \pi x), \quad \lambda_{i}=(i \pi)^{4}, \quad i=1,2,3 \ldots
$$

Let $V_{N}$ be the finite-dimensional subspace of the space $H^{2}(\Omega) \cap H_{0}^{1}(\Omega)$ spanned by the first $N$ eigenfunctions, i.e., $V_{N}=\operatorname{span}\left\{\phi_{i}\right\}_{i=1}^{N}$. The eigenfunction expansion discretization for (1.1) is as follows: find the coefficients $z_{i} \in \mathbb{R}$ in the linear combination of the $\phi_{i}$,

$$
y_{N}(x)=\sum_{i=1}^{N} z_{i} \phi_{i}(x)=\sqrt{2} \sum_{i=1}^{N} z_{i} \sin (i \pi x) \in V_{N},
$$

such that

$$
A\left(y_{N}, \phi\right)=0 \quad \forall \phi \in V_{N},
$$

where the $z_{i}$ are unknown coefficients to be determined. By substituting (2.2) into (2.1), we obtain the following equations

$$
\begin{array}{r}
(\sqrt{2})^{2} \sum_{i=1}^{N} z_{i} \int_{0}^{1}\left[(i \pi)^{2}(m \pi)^{2} \sin (i \pi x) \sin (m \pi x)-\alpha(i \pi)(m \pi) \cos (i \pi x) \cos (m \pi x)\right. \\
+\beta \sin (i \pi x) \sin (m \pi x)] d x=\sqrt{2} \int_{0}^{1}\left[f\left(y_{N}\right)+g(x)\right] \sin (i \pi x) d x .
\end{array}
$$

Since $\int_{0}^{1} \sin (i \pi x) \sin (m \pi x) d x=\int_{0}^{1} \cos (i \pi x) \cos (m \pi x) d x=\frac{1}{2} \delta_{i m}$, we obtain 


$$
z_{i}\left(i^{4} \pi^{4}-\alpha i^{2} \pi^{2}+\beta\right)=\sqrt{2} \int_{0}^{1}\left[f\left(y_{N}\right)+g(x)\right] \sin (i \pi x) d x .
$$

From the above equations, we obtain a complicated system of polynomial equations with respect to $z=\left(z_{1}, z_{2}, \ldots, z_{N}\right)$ that can be expressed as follows:

$$
\begin{aligned}
& F^{N}\left(z_{1}, z_{2}, \ldots, z_{N}\right)= \\
& \quad\left[\begin{array}{c}
z_{1} v_{1}-\int_{0}^{1} f\left(z_{1} \phi_{1}+z_{2} \phi_{2}+\cdots+z_{N} \phi_{N}\right) \phi_{1}-\int_{0}^{1} g \phi_{1} \\
z_{2} v_{2}-\int_{0}^{1} f\left(z_{1} \phi_{1}+z_{2} \phi_{2}+\cdots+z_{N} \phi_{N}\right) \phi_{2}-\int_{0}^{1} g \phi_{2} \\
\vdots \\
z_{N-1} v_{N-1}-\int_{0}^{1} f\left(z_{1} \phi_{1}+z_{2} \phi_{2}+\cdots+z_{N} \phi_{N}\right) \phi_{N-1}-\int_{0}^{1} g \phi_{N-1} \\
z_{N} v_{N}-\int_{0}^{1} f\left(z_{1} \phi_{1}+z_{2} \phi_{2}+\cdots+z_{N} \phi_{N}\right) \phi_{N}-\int_{0}^{1} g \phi_{N}
\end{array}\right]=0,
\end{aligned}
$$

where $v_{i}=\left[(i \pi)^{4}-\alpha(i \pi)^{2}+\beta\right], \quad i=1,2, \ldots, N$.

Given that $N$ is large, it is difficult to find all solutions of the system of polynomial equations in (2.4). However, the total problem can be decomposed into a series of subproblems that can be solved. Hence, we utilize some special algorithms for the resulting system of polynomial equations.

Lemma 2.1. (See [30]) For $k$ positive integers $j_{1}, j_{2}, \ldots, j_{k}$, let $c\left(j_{1}, j_{2}, \ldots, j_{k}\right)$ denote $\int_{0}^{1} \sin \left(j_{1} \pi x\right) \sin \left(j_{2} \pi x\right) \cdots \sin \left(j_{k} \pi x\right) d x$. Then, the following results hold:

(i) When $k$ is odd and $\sum_{l=1}^{k} j_{l}$ is even, $c=0$;

(ii) When $k$ and $\sum_{l=1}^{k} j_{l}$ are odd

$$
c=\frac{(-1)^{(k-1) / 2}}{\pi 2^{k-2}} \sum_{\substack{\sigma_{l} \in\{-1,1\} \\ l=2, \cdots, k}} \frac{\sigma_{2} \sigma_{3} \cdots \sigma_{k}}{j_{1}+\sigma_{2} j_{2}+\cdots+\sigma_{k} j_{k}} ;
$$

(iii) When $k$ is even,

$$
c=\frac{(-1)^{k / 2}}{2^{k-1}} \sum_{\substack{\sigma_{l} \in\{-1,1\} \\ l=2, \cdots, k}} \sigma_{2} \sigma_{3} \cdots \sigma_{k} \delta\left(j_{1}+\sigma_{2} j_{2}+\cdots+\sigma_{k} j_{k}, 0\right)
$$

where $\delta(\cdot, \cdot)$ represents the Kronecker delta.

The calculation of $\int_{0}^{1} f\left(z_{1} \phi_{1}+z_{2} \phi_{2}+\cdots+z_{N} \phi_{N}\right) \phi_{i}$ in (2.4) depends on the results from the Lemma 2.1. Then, we have

$$
\begin{aligned}
\int_{0}^{1} \phi_{j_{1}} \cdots \phi_{j_{l}} \phi_{i} d x & =\sqrt{2}^{(l+1)} \int_{0}^{1} \sin \left(r_{j_{1}} \pi x\right) \cdots \sin \left(r_{j_{k}} \pi x\right) \sin \left(r_{i} \pi x\right) d x \\
& =\sqrt{2}^{(l+1)} c\left(r_{j_{1}}, r_{j_{2}}, \ldots, r_{j_{k}}, r_{i}\right) .
\end{aligned}
$$

Accordingly, the integral (2.5) corresponding to monomial $a_{l} y_{N}^{l}$ of $f\left(y_{N}\right)$ can be obtained as follows: 


$$
\begin{aligned}
& \int_{0}^{1} a_{l}\left(z_{1} \phi_{1}+\cdots+z_{N} \phi_{N}\right)^{l} \phi_{i} d x \\
& =a_{l} \int_{0}^{1} \sum_{\substack{k_{1}+k_{2}+\cdots+k_{N}=l \\
k_{1}, k_{2}, \ldots, k_{N} \geq 0}}\left(\begin{array}{c}
l \\
k_{1}, \ldots, k_{N}
\end{array}\right)\left(z_{1} \phi_{1}\right)^{k_{1}}\left(z_{2} \phi_{2}\right)^{k_{2}} \cdots\left(z_{t} \phi_{t}\right)^{k_{N}} \phi_{i} d x \\
& =a_{l} \sum_{\substack{k_{1}+k_{2}+\cdots+k_{N}=l \\
k_{1}, k_{2}, \ldots, k_{N} \geq 0}}\left(\begin{array}{c}
l \\
k_{1}, \ldots, k_{N}
\end{array}\right) z_{1}^{k_{1}} z_{2}^{k_{2}} \cdots z_{N}^{k_{N}} \int_{0}^{1} \phi_{1}^{k_{1}} \phi_{2}^{k_{2}} \cdots \phi_{N}^{k_{N}} \phi_{i} d x \\
& =\sqrt{2}^{(l+1)} a_{l} \sum_{\substack{k_{1}+\cdots+k_{N}=l \\
k_{1}, \ldots, k_{N} \geq 0}}\left(\begin{array}{c}
l \\
k_{1}, \ldots, k_{N}
\end{array}\right) c(\underbrace{r_{1}, \ldots, r_{1}}_{k_{1}}, \underbrace{r_{2}, \ldots, r_{2}}_{k_{2}}, \ldots, \underbrace{r_{N}, \ldots, r_{N}}_{k_{N}}) z_{1}^{k_{1}} z_{2}^{k_{2}} \cdots z_{N}^{k_{N}},
\end{aligned}
$$

where $\left(\begin{array}{c}l \\ k_{1}, \ldots, k_{N}\end{array}\right)=\frac{l !}{k_{1} ! k_{2} ! \ldots \ldots k_{N} !}$ denotes a multinomial coefficient. The integral $\int_{0}^{1} g(x) \phi_{i} d x$ may not be calculated explicitly. So we can calculate it approximately by using the numerical integration (quadrature) rules. Combining all these results, the integrals in (2.4) are obtained, therefore the resulting system of polynomial equations is:

$$
F^{N}\left(z_{1}, z_{2}, \ldots, z_{N}\right)=\left[\begin{array}{c}
\sum_{|j|=0}^{d} c_{j}^{(1)} z^{j} \\
\sum_{|j|=0}^{d} c_{j}^{(2)} z^{j} \\
\vdots \\
\sum_{|j|=0}^{d} c_{j}^{(N)} z^{j}
\end{array}\right]=0,
$$

where $j=\left(j_{1}, \ldots, j_{N}\right),|j|=j_{1}+\cdots+j_{N}, z^{j}=z_{1}^{j_{1}} z_{2}^{j_{2}} \cdots z_{N}^{j_{N}}$, and $c_{j}^{k}$ is the corresponding coefficient in component $k$ of $F^{N}$.

3. Construction of the extension homotopy for the system of polynomial equations. Some numerical methods have been proposed to deal with the algebraic sets generated from the system of polynomial equations. Basic numerical algebraic geometry provides the algorithms with probability one while computing all isolated solutions of a system of polynomial equations. In this paper, we consider the homotopy defined by;

$$
H^{N}\left(z_{1}, z_{2}, \ldots, z_{N}, t\right)=t G^{N}\left(z_{1}, z_{2}, \ldots, z_{N}\right)+(1-t) F^{N}\left(z_{1}, z_{2}, \ldots, z_{N}\right),
$$

where $F^{N}$ is defined in (2.4) and $G^{N}$ is defined as follows:

$$
\begin{aligned}
& G^{N}\left(z_{1}, z_{2}, \ldots, z_{N}\right) \triangleq \\
& \quad\left[\begin{array}{c}
z_{1} v_{1}-\int_{0}^{1} f\left(z_{1} \phi_{1}+z_{2} \phi_{2}+\cdots+z_{N-1} \phi_{N-1}\right) \phi_{1}-\int_{0}^{1} g \phi_{1} \\
z_{2} v_{2}-\int_{0}^{1} f\left(z_{1} \phi_{1}+z_{2} \phi_{2}+\cdots+z_{N-1} \phi_{N-1}\right) \phi_{2}-\int_{0}^{1} g \phi_{2} \\
\vdots \\
z_{N-1} v_{N-1}-\int_{0}^{1} f\left(z_{1} \phi_{1}+z_{2} \phi_{2}+\cdots+z_{N-1} \phi_{N-1}\right) \phi_{N-1}-\int_{0}^{1} g \phi_{N-1} \\
z_{N} v_{N}-\int_{0}^{1} f\left(z_{1} \phi_{1}+z_{2} \phi_{2}+\cdots+z_{N} \phi_{N}\right) \phi_{N}-\int_{0}^{1} g \phi_{N}
\end{array}\right] .
\end{aligned}
$$


The case $G^{N}=0$ corresponds to the variational problem: find $\bar{y}_{N}=\sum_{i=1}^{N} z_{i} \phi_{i} \in V_{N}$, such that

$$
\begin{aligned}
& \int_{0}^{1}\left[\left(P_{N-1} \bar{y}_{N}\right)^{\prime \prime} \phi^{\prime \prime}-\alpha\left(P_{N-1} \bar{y}_{N}\right)^{\prime} \phi^{\prime}+\beta\left(P_{N-1} \bar{y}_{N}\right) \phi\right] d x-\int_{0}^{1} f\left(P_{N-1} \bar{y}_{N}\right) d x \\
& -\int_{0}^{1} g \phi d x=0 \quad \forall \phi \in V_{N-1} \\
& \int_{0}^{1}\left[\bar{y}_{N}^{\prime \prime} \phi_{N}^{\prime \prime}-\alpha \bar{y}_{N}^{\prime} \phi_{N}^{\prime}+\beta \bar{y}_{N} \phi_{N}\right] d x-\int_{0}^{1} f\left(\bar{y}_{N}\right)-\int_{0}^{1} g \phi_{N} d x=0
\end{aligned}
$$

where $P_{N-1}: V \longrightarrow V_{N-1}$ denotes to the orthogonal projection that is defined as: for $y \in V$

$$
\left(y-P_{N-1} y, \phi\right)=0 \quad \forall \phi \in V_{N-1},
$$

where $(\cdot, \cdot)$ is the $L^{2}$ inner product on the domain $\Omega$.

The starting system of the homotopy is $H^{N}\left(z_{1}, \ldots, z_{N}, 1\right)=G^{N}\left(z_{1}, \ldots, z_{N}\right)=0$ and the target system is $H^{N}\left(z_{1}, \ldots, z_{N}, 0\right)=F^{N}\left(z_{1}, \ldots, z_{N}\right)=0$. Therefore, we expect that the path-tracking (path-following) for the homotopy equation $H^{N}\left(z_{1}, \ldots, z_{N}, t\right)=0$ is efficient, since $F^{N}$ and $G^{N}$ have similar structures. The computational cost for the target system is divided into two parts. The first part is the cost of tracking the homotopy paths defined by $H^{N}=0$ and the second part is the computation of starting points $S_{N}$ of the paths defined by $G^{N}=0$. In this paper, we get the starting points $S_{N}$ of the homotopy paths by solving $F^{N-1}\left(z_{1}, \ldots, z_{N-1}\right)=0$ and use the solution $\left(z_{1}, \ldots, z_{N-1}\right)^{\prime}$ in the last equation to calculate the last components $z_{N}$. We repeat the same technique again to get a recursive process. Therefore, the solution of the target system $F^{N}=0$ is obtained by successively tracking the paths $H^{n}=0$ and solving the last equations of $G^{n}=0$, with $n=2, \ldots, N$.

The key feature and the basic tool in homotopy continuation are path-tracking. However, path-tracking often encounters difficulty when the path is singular as $t$ varies from 0 to 1 , where singularity means that the Jacobian matrix has rank less than $n$. To tackle this problem, a generic random number $\gamma \in \mathbb{C}$ (the accessibility constant) is used [20]. Therefore, we extend the homotopy for a system of polynomial equations as

$$
H^{n}\left(z_{1}, \ldots, z_{n}, t\right)=\gamma t G^{n}\left(z_{1}, \ldots, z_{n}\right)+(1-t) F^{n}\left(z_{1}, \ldots, z_{n}\right), \quad n=2, \ldots, N
$$

with the following Algorithm 1:

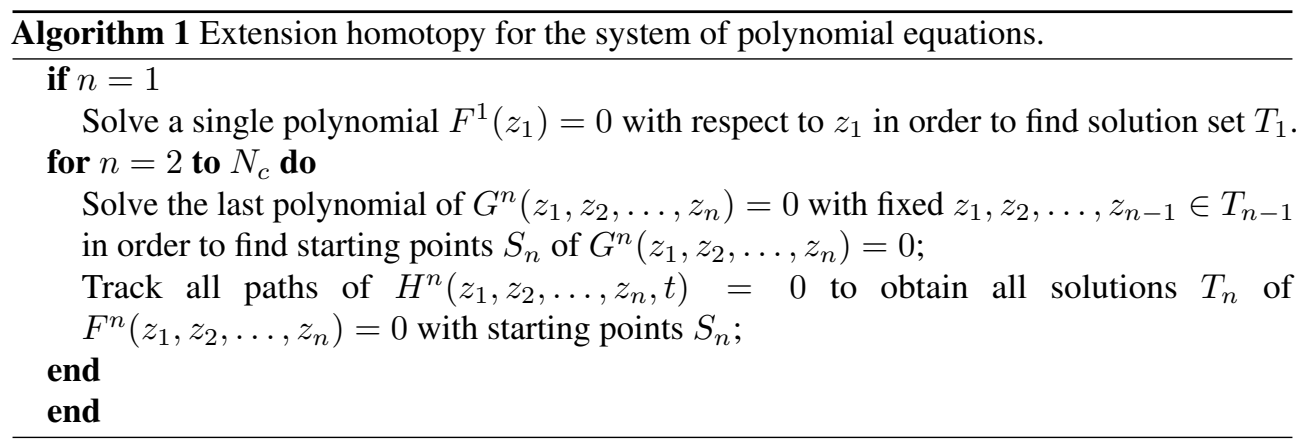

The homotopy (3.1) is guaranteed to obtain all solutions of $F^{n}=0$ which arise from the discretization of a differential equation if $\operatorname{deg}(f(y))$ is odd, under certain assumptions [24]. But when $\operatorname{deg}(f(y))$ is even, we can not guarantee that the homotopy obtains all solutions of $F^{n}=0$. If desired, we can refine all solutions obtained with a nonlinear solver. 
4. Error estimates for eigenfunction expansion method. We introduce some notations that are needed throughout this section. Given that $s$ is a nonnegative integer, let $\dot{H}^{s}=\dot{H}^{s}(\Omega)$ be a subspace of $L^{2}(\Omega)$ consisting of functions $v$, with $|v|_{s}=\left(\sum_{m=1}^{\infty} \lambda_{m}^{s}\left(v, \phi_{m}\right)^{2}\right)^{\frac{1}{2}}<\infty$. It is known that $\dot{H}^{s}(\Omega)=\left\{y \in H^{s}, y^{(2 j)}(0)=y^{(2 j)}(1)=0,0 \leq j<s / 2\right\}$, and the norms $|\cdot|_{s}$ and $\|\cdot\|_{s}$ are equivalent in $\dot{H}^{s}(\Omega)$; see [25].

Consider a nonlinear differential operator $\mathcal{L}$ defined in $H^{2}(\Omega) \cap H_{0}^{1}(\Omega)$ as follows,

$$
\mathcal{L}(w)=w^{(4)}+\alpha w^{\prime \prime}+\beta w-f(w)-g(x) .
$$

The linearized operator of $\mathcal{L}$ at $w$ (Frechet derivative) is then given by,

$$
\mathcal{L}^{\prime}(w) v=v^{(4)}+\alpha v^{\prime \prime}+\beta v-f^{\prime}(w) v .
$$

We assume that $y$ is a solution of $(1.1)$ and $\mathcal{L}^{\prime}(y)$ is an isomorphism from $V(\Omega)$ to $V^{*}(\Omega)$. Define the bilinear form $A^{\prime}(\cdot ; \cdot, \cdot): V \times V \longrightarrow \mathbb{R}$ as follows

$$
A^{\prime}(w ; v, \phi)=\int_{0}^{1}\left(v^{\prime \prime} \phi^{\prime \prime}-\alpha v^{\prime} \phi^{\prime}+\beta v \phi\right) d x-\int_{0}^{1} f^{\prime}(w) v \phi d x
$$

Let $P_{N}$ be the $L^{2}$-orthogonal projection defined by

$$
\left(v-P_{N} v, \phi\right)=0, \quad \forall \phi \in V_{N}
$$

and define the Ritz projection (elliptic projection) $A^{\prime}(y ; \cdot, \cdot)$ such that

$$
A^{\prime}\left(y ; v-R_{N} v, \phi\right)=0, \quad \forall \phi \in V_{N} .
$$

We present some lemmas which will help us to clarify the proofs of our main results.

LEMMA 4.1. Let $v \in \dot{H}^{s+1}(\Omega)$, for $s \geq 1$. The following error estimates for the projection $P_{N}$ hold

$$
\left\|v-P_{N} v\right\|_{H^{1}} \leq C N^{-s}|v|_{1+s}, \quad\left\|v-P_{N} v\right\|_{L^{2}} \leq C N^{-(s+1)}|v|_{1+s},
$$

where $C$ stands for a generic positive constant which is independent of $N$.

For the proof of Lemma 4.1; see [8].

Lemma 4.2. Consider y the solution of problem (2.1). For $v_{N} \in V_{N}$ and $N$ sufficiently large, we have

$$
\left\|v_{N}\right\|_{H^{1}} \leq C \sup _{\phi \in V_{N}} \frac{A^{\prime}\left(y ; v_{N}, \phi\right)}{\|\phi\|_{H^{1}}} \quad \text { and } \quad\left\|v_{N}\right\|_{H^{1}} \leq C \sup _{\phi \in V_{N}} \frac{A^{\prime}\left(y ; \phi, v_{N}\right)}{\|\phi\|_{H^{1}}}
$$

The same results are also valid for $\varepsilon$ sufficiently small when replacing $A^{\prime}(y ; \cdot, \cdot)$ in (4.1) by $A^{\prime}\left(y_{\varepsilon} ; \cdot, \cdot\right)$ with $\left\|y_{\varepsilon}-y\right\|_{H^{1}} \leq \varepsilon$.

Proof. Under the assumption that $\mathcal{L}^{\prime}(y)$ is an isomorphism, we get

$$
\left\|v_{N}\right\|_{H^{1}} \leq C \sup _{\phi \in V} \frac{A^{\prime}\left(y ; v_{N}, \phi\right)}{\|\phi\|_{H^{1}}}
$$


Based on the definition of $P_{N}$ and Sobolev's embedding theorem for a one-dimensional domain $\Omega, H^{1}(\Omega) \hookrightarrow L^{p}(\Omega), 1 \leq p<+\infty$, we have

$$
\begin{aligned}
A^{\prime}\left(y ; v_{N}, w-P_{N} w\right) & =\int_{0}^{1}\left[v_{N}^{\prime \prime}\left(w-P_{N} w\right)^{\prime \prime}-\alpha v_{N}^{\prime}\left(w-P_{N} w\right)^{\prime}+\beta v_{N}\left(w-P_{N} w\right)\right] d x \\
& -\int_{0}^{1} f^{\prime}(y) v_{N}\left(w-P_{N} w\right) d x \\
& =\int_{0}^{1}\left[v_{N}^{\prime \prime \prime \prime}\left(w-P_{N} w\right)-\alpha v_{N}^{\prime \prime}\left(w-P_{N} w\right)+\beta v_{N}\left(w-P_{N} w\right)\right] d x \\
& -\int_{0}^{1} f^{\prime}(y) v_{N}\left(w-P_{N} w\right) d x \\
& =-\int_{0}^{1} f^{\prime}(y) v_{N}\left(w-P_{N} w\right) d x .
\end{aligned}
$$

Using Hölder's inequality and Lemma 4.1, we therefore obtain

$$
A^{\prime}\left(y ; v_{N}, w-P_{N} w\right) \leq\left\|f^{\prime}(y)\right\|_{L^{4}}\left\|v_{N}\right\|_{L^{4}}\left\|w-P_{N} w\right\|_{L^{2}} \leq C N^{-1}\|w\|_{H^{1}}\left\|v_{N}\right\|_{H^{1}} .
$$

Then

$$
\begin{aligned}
A^{\prime}\left(y ; v_{N}, P_{N} w\right) & =A^{\prime}\left(y, v_{N}, w\right)-A^{\prime}\left(y, v_{N}, w-P_{N} w\right) \\
& \geq A^{\prime}\left(y, v_{N}, w\right)-C N^{-1}\|w\|_{H^{1}}\left\|v_{N}\right\|_{H^{1}} .
\end{aligned}
$$

Therefore, the first estimate in (4.1) follows through the use of $\left\|P_{N} w\right\|_{H^{1}} \leq\|w\|_{H^{1}}$. Similarly, we can show the second estimate.

Again for $A^{\prime}\left(y_{\varepsilon} ; \cdot, \cdot\right)$, we use Sobolev's embedding theorem and Hölder's inequality. Therefore, we get

$$
\begin{aligned}
& A^{\prime}\left(y_{\varepsilon} ; v, \phi\right)=\int_{0}^{1}\left[v^{\prime \prime} \phi^{\prime \prime}-\alpha v^{\prime} \phi^{\prime}+\beta v \phi\right] d x-\int_{0}^{1} f^{\prime}\left(y_{\varepsilon}\right) v \phi d x \\
& \quad=\int_{0}^{1}\left[v^{\prime \prime} \phi^{\prime \prime}-\alpha v^{\prime} \phi^{\prime}+\beta v \phi\right] d x-\int_{0}^{1} f^{\prime}(y) v \phi d x+\int_{0}^{1}\left[f^{\prime}(y)-f^{\prime}\left(y_{\varepsilon}\right)\right] v \phi d x .
\end{aligned}
$$

By using the Taylor expansion with the integral remainder over the interval $\Omega$, we have

$$
\begin{aligned}
A^{\prime}\left(y_{\varepsilon} ; v, \phi\right) & =A^{\prime}(y ; v, \phi)-\int_{0}^{1}\left[f^{\prime \prime}(y)\left(y_{\varepsilon}-y\right)+\cdots+\frac{f^{(d)}(y)}{(d-1) !}\left(y_{\varepsilon}-y\right)^{d-1}\right] v \phi d x \\
& \geq A^{\prime}(y ; v, \phi)-C\left\|\left(y_{\varepsilon}-y\right)\right\|_{H^{1}}\|v\|_{H^{1}}\|\phi\|_{H^{1}} .
\end{aligned}
$$

The proof is completed.

LEMMA 4.3. The following formula holds for the difference between the solution y and its Ritz projection $R_{N} y$.

$$
\left\|y-R_{N} y\right\|_{L^{2}} \leq C N^{-1}\left\|y-R_{N} y\right\|_{H^{1}} .
$$

Proof. Let $e_{N}=y-R_{N} y$ and define $z$ as the solution of the following equation,

$$
\begin{aligned}
A^{\prime}(y ; z, \phi) & \triangleq \int_{0}^{1}\left(z^{\prime \prime} \phi^{\prime \prime}-\alpha z^{\prime} \phi^{\prime}+\beta z \phi\right) d x-\int_{0}^{1} f^{\prime}(y) z \phi d x \\
& =\left(e_{N}, \phi\right), \quad \forall \phi \in V .
\end{aligned}
$$


Setting $\phi=e_{N}$ in (4.2) we obtain

$$
\begin{aligned}
\left\|e_{N}\right\|_{L^{2}}^{2} & =A^{\prime}\left(y ; z, e_{N}\right) \\
& =A^{\prime}\left(y ; z-P_{N} z, e_{N}\right) \\
& \leq C\left\|z-P_{N} z\right\|_{H^{1}}\left\|e_{N}\right\|_{H^{1}} \\
& \leq C N^{-1}|z|_{2}\left\|e_{N}\right\|_{H^{1}} .
\end{aligned}
$$

Since the norms $|\cdot|_{2}$ and $\|\cdot\|_{H^{2}}$ are equivalent and (4.2) satisfy the regularity condition $\|z\|_{H^{2}} \leq C\left\|e_{N}\right\|_{L^{2}}$, we have

$$
\left\|e_{N}\right\|_{L^{2}} \leq C N^{-1}\left\|e_{N}\right\|_{H^{1}} .
$$

THEOREM 4.4. Let $y \in \dot{H}^{s+1}$ be the solution of (2.1) and $y_{N} \in V_{N}$ be the approximate solution of (2.3). The following error estimates hold, if $N$ is sufficiently large.

$$
\left\|y_{N}-y\right\|_{H^{1}} \leq C N^{-s}, \quad\left\|y_{N}-y\right\|_{L^{2}} \leq C N^{-(s+1)} .
$$

Proof. The proof follows a similar argument as [31].

5. Filters for removing spurious solutions. The discretized boundary value problems sometimes have spurious solutions that are not reasonable approximate solutions to the original equation. Therefore, we have to remove them. In this section, we present two types of filters for removing spurious solutions of the nonlinear fourth-order boundary value problem. The first type depends on the finite difference approximation, which has two forms, and the second type depends on Newton's method.

For the first form of the finite difference filter, we apply the central finite differences to obtain a discretization of the nonlinear fourth-order boundary value problem. For larger N, discard $y \in V_{N}$ for which the residual is large, i.e., if,

$$
\begin{array}{r}
\sum_{i=3}^{N-1} \mid \frac{y_{i+2}-4 y_{i+1}+6 y_{i}-4 y_{i-1}+y_{i-2}}{h^{4}}+\alpha \frac{y_{i+1}-2 y_{i+1}+y_{i-1}}{h^{2}}+\beta y_{i}- \\
\quad f\left(y_{i}\right)-g\left(x_{i}\right) \mid>\varepsilon
\end{array}
$$

where $\varepsilon>0$ is a prescribed parameter.

For the second form of the finite difference filter, we take the derivative of the original differential equation and obtain $y^{(5)}+\alpha y^{\prime \prime \prime}+\beta y^{\prime}=y^{\prime} f^{\prime}\left(y_{i}\right)+g^{\prime}\left(x_{i}\right)$, and then discretize it by a finite difference method. We throw away those solutions $y \in V_{N}$ of EEM for which,

$$
\begin{array}{r}
\sum_{i=4}^{N-2} \mid \frac{y_{i+3}-4 y_{i+2}+5 y_{i+1}-5 y_{i-1}+4 y_{i-2}-y_{i-3}}{2 h^{5}}+\alpha \frac{y_{i+2}-2 y_{i+1}+2 y_{i-1}-y_{i-2}}{2 h^{3}} \\
+\beta \frac{y_{i+1}-y_{i-1}}{2 h}-\frac{y_{i+1}-y_{i-1}}{2 h} f^{\prime}\left(y_{i}\right)+g^{\prime}\left(x_{i}\right) \mid>\varepsilon,
\end{array}
$$

where $\varepsilon>0$ again is a prescribed parameter.

Let $y$ be a solution of the nonlinear fourth-order boundary value problem and suppose that the finite difference discretization has the following error estimate for some $r>0$,

$$
\left\|y-y_{h}\right\| \leq C h^{r},
$$


where the constant $C$ depends on derivatives of the exact solution $y$. Therefore, if $y$ contains some modes with more oscillations, the corresponding $C$ will be large. This observation provides some hints for choosing $\varepsilon$. If $\varepsilon$ is small, then only solutions with few oscillations will remain; if $\varepsilon$ is larger, then solutions with many oscillations will also remain. We illustrate the variations of peaks by numerical examples.

The second filter depends on the Newton method that relates to the error estimate for the eigenfunction expansion discretization, as mentioned in Section 4. The approximate solutions of the discretized problems on successively finer levels satisfy $\left\|y-y_{N}\right\|_{H^{1}} \leq C N^{-s}$, $\left\|y-y_{N}\right\|_{L^{2}} \leq C N^{-(s+1)}$, and can be viewed as a solution path $y(x ; N)$ parameterized by the discretization level $N$. Therefore, the true approximate solutions should lie on a solution path. For larger $N$, the Cauchy criterion for convergence implies that $\left\|y_{N}-y_{N+1}\right\|$ is very small. While applying Newton's method to the discretization system $A\left(y_{N}, \phi\right)=0, \forall \phi \in V_{N}$, with initial guesses $y_{N}=y_{N-1}$, the Newton method is expected to converge to nonspurious solutions. In Section 6, we present numerical evidence to support our filters.

6. Numerical results and discussions. In this section, we will verify the efficiency of the extension homotopy and accuracy of the EEM.

6.1. Efficiency of the extension homotopy. Numerical experiments have been performed on the boundary value problem (1.1) with $\alpha=\beta=1$, the polynomial $f(y)=y^{3}$, and the function $g(x)=\pi^{4} \sin (\pi x)-\pi^{2} \sin (\pi x)+\sin (\pi x)+\sin (\pi x)-\sin ^{3}(\pi x)$. Since the PHC package provides the option to accept a user-defined start system, we verify the efficiency of the extension homotopy with the help of PHC. At every intermediate $n$, we call the PHC package three times: First for solving the target system by the total degree homotopy; secondly, for obtaining starting points while solving the start system by the total degree homotopy and, lastly, for tracking the paths with the starting points obtained from the second call.

EXAMPLE 6.1. Consider the following nonlinear fourth-order boundary value problem with boundary conditions

$$
\begin{aligned}
y^{(4)}+y^{\prime \prime}+y & =y^{3}+\pi^{4} \sin (\pi x)-\pi^{2} \sin (\pi x)+\sin (\pi x)+\sin (\pi x)-\sin ^{3}(\pi x) \\
y(0) & =y(1)=y^{\prime \prime}(0)=y^{\prime \prime}(1)=0
\end{aligned}
$$

The boundary value problem (6.1) may have infinitely many solutions [9], and the discretized problem has finitely many solutions. Table 6.1 lists the solution data for the discretized problem (6.1) in the subspace $V_{10}$. The first part of Table 6.1 contains the solutions of system of polynomial equations by the total degree homotopy. The last part consists of the solutions of system of polynomial equations using the extension homotopy by tracking the paths of $H^{n}=0$ with known start solutions $S_{n}, n=2, \ldots, N$. The \# Regular sols refers to the numbers of regular solutions of the system of polynomial equations, including the complex solutions. The number of regular solution is increasing with respect to the number of eigenfunctions $n$. Likewise, the \# Real sols refers to the number of real solutions, which have zero imaginary parts, obtained after solving the target system of polynomial equations by these two homotopies.

From Table 6.1, in the first part it takes 3 hours and 12 minutes for the total degree homotopy when $n=10$, while the time taken in the second part (the extension homotopy) is 1 hour and 26 minutes. Comparing the runtime one can observe a time difference of 1 hour and 37 minutes. In fact, the random number in homotopy continuation was considered in (3.1) as the reason why the numbers of regular solutions in the second columns of I and II of Table 6.1 may be slightly different. Similarly, there is little difference in the numbers of the real solutions; see the third columns of I and II of Table 6.1. 


\section{ETNA}

Kent State University and

Johann Radon Institute (RICAM)

TABLE 6.1

The solution data for the discretized problem (6.1) in $V_{10}$.

I. Total degree homotopy

\begin{tabular}{lrcr}
\hline $\mathrm{n}$ & \# Regular sols & \# Real sols & Time \\
\hline 1 & 3 & 3 & $\sim 0 \mathrm{~ms}$ \\
2 & 9 & 5 & $10 \mathrm{~s} 711 \mathrm{~ms}$ \\
3 & 27 & 7 & $15 \mathrm{~s} 203 \mathrm{~ms}$ \\
4 & 81 & 9 & $12 \mathrm{~s} 919 \mathrm{~ms}$ \\
5 & 243 & 11 & $13 \mathrm{~s} 876 \mathrm{~ms}$ \\
6 & 729 & 13 & $24 \mathrm{~s} 747 \mathrm{~ms}$ \\
7 & 2187 & 15 & $1 \mathrm{~m} 31 \mathrm{~s} 975 \mathrm{~ms}$ \\
8 & 6560 & 17 & $7 \mathrm{~m} 44 \mathrm{~s} 297 \mathrm{~ms}$ \\
9 & 19679 & 19 & $37 \mathrm{~m} 14 \mathrm{~s} 434 \mathrm{~ms}$ \\
10 & 59033 & 21 & $3 \mathrm{~h} 12 \mathrm{~m} 45 \mathrm{~s} 206 \mathrm{~ms}$
\end{tabular}

II. Tracking the paths of $H^{n}$ with known $S_{n}$

\begin{tabular}{lrrr}
\hline $\mathrm{n}$ & \# Regular sols & \# Real sols & Time \\
\hline 1 & & & \\
2 & 9 & 5 & $1 \mathrm{~ms}$ \\
3 & 27 & 7 & $29 \mathrm{~ms}$ \\
4 & 81 & 9 & $139 \mathrm{~ms}$ \\
5 & 243 & 11 & $1 \mathrm{~s} 64 \mathrm{~ms}$ \\
6 & 729 & 13 & $6 s 268 \mathrm{~ms}$ \\
7 & 2181 & 15 & $39 \mathrm{~s} 662 \mathrm{~ms}$ \\
8 & 6561 & 17 & $3 \mathrm{~m} 31 \mathrm{~s} 953 \mathrm{~ms}$ \\
9 & 19679 & 19 & $16 \mathrm{~m} 31 \mathrm{~s} 230 \mathrm{~ms}$ \\
10 & 59014 & 25 & $1 \mathrm{~h} 26 \mathrm{~m} 2 \mathrm{~s} 262 \mathrm{~ms}$
\end{tabular}

As we know spurious solutions may appear when solving problem (6.1). Then, it is necessary to use the filters mentioned in Section 5 to remove the possible spurious solutions. After obtaining the solutions for $n=13$ from HOM4PS-2.0, we apply both the finite difference filter and the Newton filter. For the Newton filter successive applications of Newton's method to systems of $n \leq 18$ were used. Table 6.2 contains the results for problem (6.1). The non-spurious solutions are shown in Figures 6.1 and 6.2. For the finite difference filter, $\varepsilon$ was chosen as $1 \mathrm{e}+3$ and $1 \mathrm{e}+6$, see Figures 6.3 and 6.4. Note that, we applied the finite difference filter with $n=13$ because of the very high cost of using HOM4PS-2.0.

As mentioned in Section 5, the finite difference filter filters solutions depending on their shape and depending on $\varepsilon$. If $\varepsilon$ is chosen small, the set of filtered solutions only contain some solutions of low oscillation. If $\varepsilon$ is chosen larger, solutions with higher oscillation will enter the filtered solution set.

6.2. Convergence rate for EEM. In order to determine the order of accuracy (convergence rate) for EEM, we take into consideration the regularity of the solution of the original problem. First, we calculate errors when solving the problem and then we get the convergence 
TABLE 6.2

The number of solutions after removing spurious solutions.
FD-filter with
FD-filter without
derivative original ODE derivative original ODE
Newton's filter

\begin{tabular}{lccc}
\hline $\mathrm{n}$ & Real solutions by HOM4PS-2.0 & Solutions after Filter(18) \\
\hline 11 & 5 & 5 & 13 \\
12 & 5 & 5 & 13 \\
13 & 5 & 5 & 11 \\
14 & - & - & 11 \\
15 & - & - & 11 \\
16 & - & - & 11 \\
17 & - & - & 11 \\
18 & - & - & 9
\end{tabular}
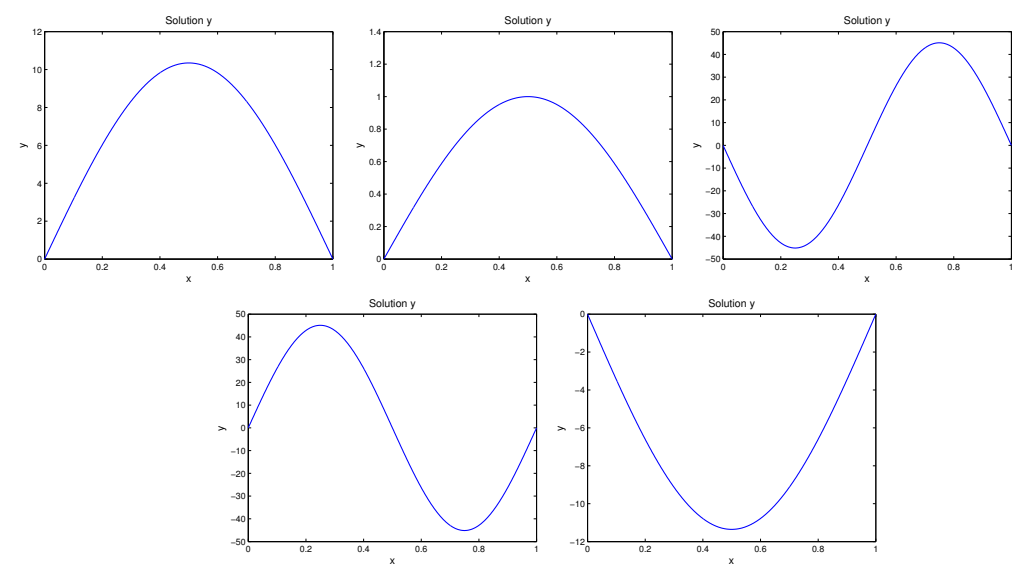

FIG. 6.1. Solution set for problem (6.1) by applying FD-filter without derivative original ODE with $n=13$ and $\varepsilon=1 \mathrm{e}+02$.

order.

EXAMPLE 6.2. Consider the following nonlinear fourth-order boundary value problem with boundary conditions

$$
\begin{gathered}
y^{(4)}+y^{\prime \prime}+y=y^{3}+g(x), \quad x \in \Omega \equiv[0,1], \\
y(0)=y(1)=y^{\prime \prime}(0)=y^{\prime \prime}(1)=0,
\end{gathered}
$$

where $g_{1}(x)$ is given such that $y_{1}=x^{3}(x-1)^{3} \sin (\pi x)$ is a solution of problem (6.2). The details of the convergence rate are shown in Table 6.3 and Figure 6.5. Note that the convergence order reaches 2 and 3.8 for the $H^{1}$-error and the $L^{2}$-error, respectively.

7. Conclusions. The eigenfunction expansion method is a suitable choice of discretization method for finding multiple solutions of fourth-order ODEs with cubic polynomial nonlinearity. The error estimates of the eigenfunction expansion method for ODEs are different from that for PDEs. The discretized system of polynomial equations is solved efficiently by the extension homotopy method. This method guarantees to find all solutions of the discretized system of polynomial equations with probability one. The Newton filter and finite difference filter are two reasonable strategies for removing possible spurious solutions. 

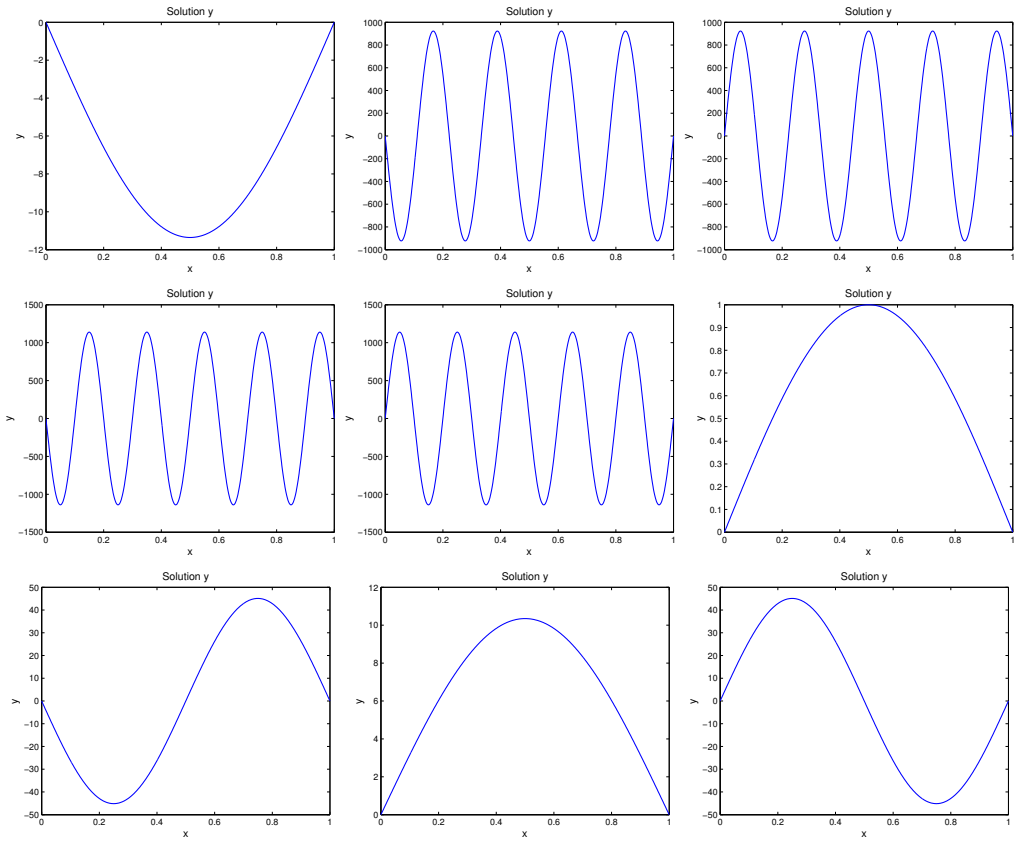

FIG. 6.2. Solution set for problem (6.1) by applying Newton's filter with $n=18$.
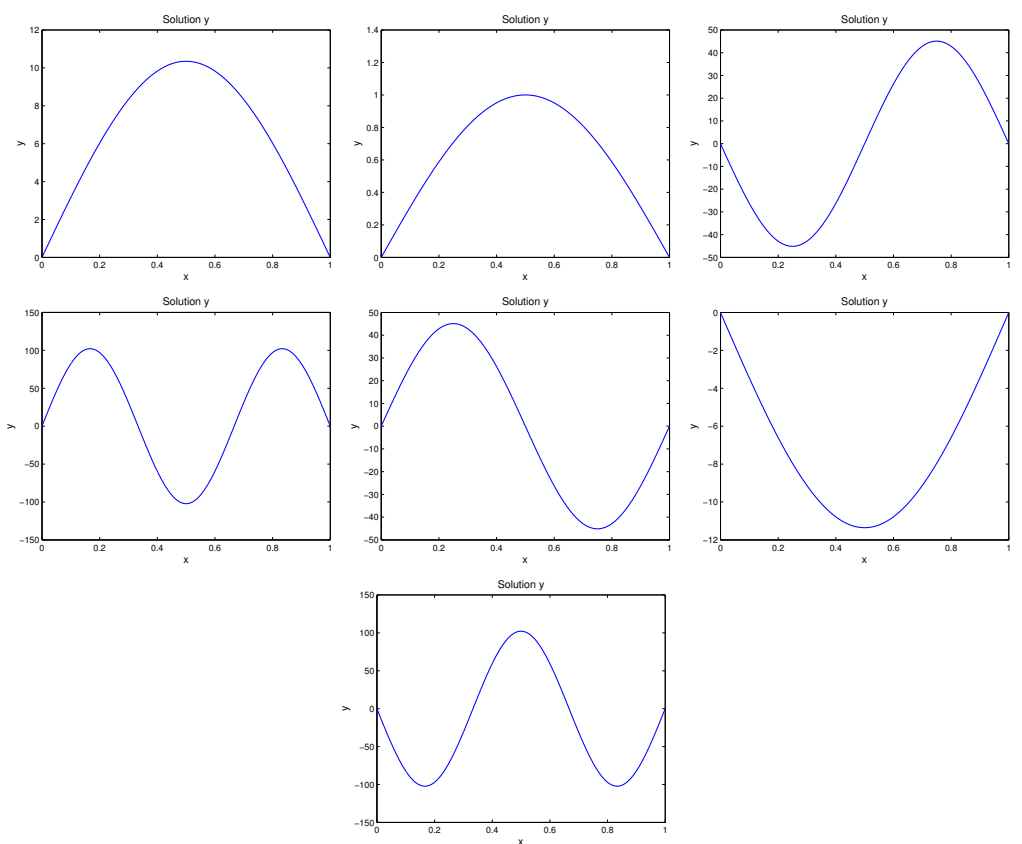

FIG. 6.3. Solution set for problem (6.1) with $\varepsilon=1 \mathrm{e}+03$. 

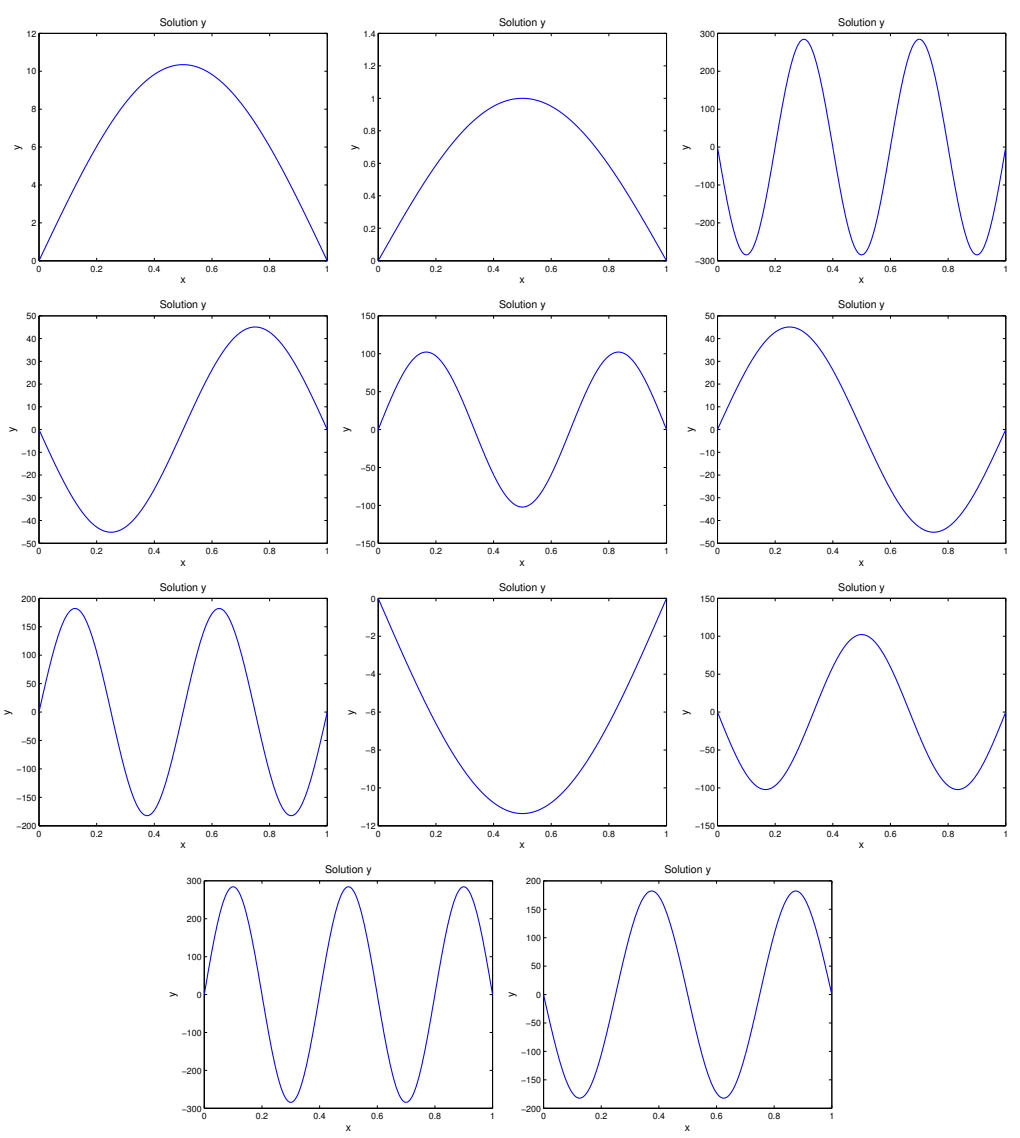

FIG. 6.4. Solution set for problem (6.1) with $\varepsilon=1 e+06$.

Acknowledgments. This work was supported in part by the National Natural Science Foundation of China $(11571061,11401075)$ and in part by the Fundamental Research Funds for the Central Universities (DUT16LK36).

\section{REFERENCES}

[1] E. L. Allgower, D. J. Bates, A. J. Sommese, And C. W. Wampler, Solution of polynomial systems derived from differential equations, Computing, 76 (2006), pp. 1-10.

[2] Z. BAI AND H. WANG, On positive solutions of some nonlinear fourth-order beam equations, J. Math. Anal. Appl., 270 (2002), pp. 357-368.

[3] D. J. Bates, J. D. Hauenstein, A. J. Sommese, and C. W. Wampler, Bertini: Software for numerical algebraic geometry, Software, 2006, available at http: / / bertini.nd.edu.

[4] G. Bonanno AND B. Di Bella, A boundary value problem for fourth-order elastic beam equations, J. Math. Anal. Appl., 343 (2008), pp. 1166-1176.

[5] - Infinitely many solutions for a fourth-order elastic beam equation, NoDEA Nonlinear Differential Equations Appl., 18 (2011), pp. 357-368.

[6] T. D. BUi AND R. A. USMANI, Solving boundary value problems in plate deflection theory, Simulation, 37 (1981), pp. 195-206.

[7] C. CHEN AND Z. XIE, Search extension method for multiple solutions of a nonlinear problem, Comput. Math. Appl., 47 (2004), pp. 327-343.

[8] - Analysis of search-extension method for finding multiple solutions of nonlinear problem, Sci. China Ser. A, 51 (2008), pp. 42-54.

[9] L. Collatz, Differential Equations: An Introduction with Applications, Wiley, 1986. 
TABLE 6.3

The rate of convergence for nonlinear fourth-order BVP (6.2).

\begin{tabular}{ccc}
$\mathrm{N}$ & $H^{1}$-error & $L^{2}$-error \\
\hline 4 & 0.0129 & $1.5984 \mathrm{e}-04$ \\
5 & 0.0063 & $3.8536 \mathrm{e}-05$ \\
6 & 0.0063 & $3.8536 \mathrm{e}-05$ \\
7 & 0.0035 & $1.2010 \mathrm{e}-05$ \\
8 & 0.0035 & $1.2010 \mathrm{e}-05$ \\
9 & 0.0022 & $4.6684 \mathrm{e}-06$ \\
10 & 0.0022 & $4.6684 \mathrm{e}-06$ \\
\hline Conv. Ord & 1.9112 & 3.8177
\end{tabular}

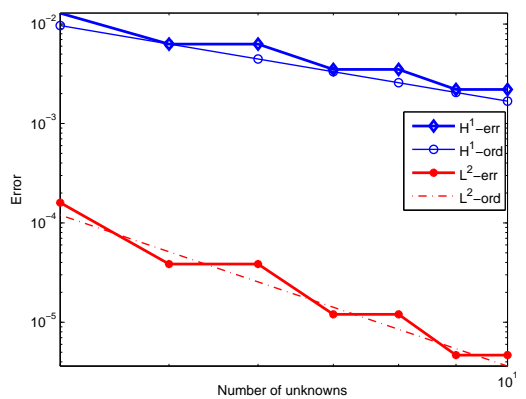

FIG. 6.5. Convergence rate for problem (6.2).

[10] M. D. R. Grossinho, L. SANChEZ, AND S. A. TERsian, On the solvability of a boundary value problem for a fourth-order ordinary differential equation, Appl. Math. Lett., 18 (2005), pp. 439-444.

[11] S. R. Gunakala, D. Comissiong, K. Jordan, AND A. SANKar, A finite element solution of the beam equation via matlab, Int. J. Appl. Sci. Tech., 2 (2012), pp. 80-88.

[12] T. Gunji, S. Kim, M. Kojima, A. Takeda, K. Fujisawa, And T. Mizutani, PHoM-a polyhedral homotopy continuation method for polynomial systems, Computing, 73 (2004), pp. 57-77.

[13] T. GyUlOV AND S. TERSIAN, Existence of trivial and nontrivial solutions of fourth-order ordinary differential equation, C. R. Acad. Bulgare Sci., 57 (2004), pp. 23-28.

[14] G. HAN AND Z. XU, Multiple solutions of some nonlinear fourth-order beam equations, Nonlinear Anal., 68 (2008), pp. 3646-3656.

[15] T. L. LEE, T. Y. LI, AND C. H. TSAI, HOM4PS-2.0: a software package for solving polynomial systems by the polyhedral homotopy continuation method, Computing, 83 (2008), pp. 109-133.

[16] T.-Y. LI, Solving polynomial systems by polyhedral homotopies, Taiwanese J. Math., 3 (1999), pp. 251-279.

[17] S. LIANG AND D. J. JEFFREY, An efficient analytical approach for solving fourth order boundary value problems, Comput. Phys. Comm., 180 (2009), pp. 2034-2040.

[18] X.-L. LIU AND W.-T. LI, Existence and multiplicity of solutions for fourth-order boundary value problems with three parameters, Math. Comput. Modelling, 46 (2007), pp. 525-534.

[19] S. T. MOHYUD-DIN AND M. A. Noor, Homotopy perturbation method for solving fourth-order boundary value problems, Math. Probl. Eng., (2007), Art. ID 98602, 15 pages.

[20] A. Morgan, Solving Polynomial Systems Using Continuation for Engineering and Scientific Problems, SIAM, Philadelphia, 2009.

[21] C. PANG, W. Dong, AND Z. WEI, Multiple solutions for fourth-order boundary value problem, J. Math. Anal. Appl., 314 (2006), pp. 464-476.

[22] M. RUYUn, Z. Jihui, AND F. ShENGMAO, The method of lower and upper solutions for fourth-order two-point boundary value problems, J. Math. Anal. Appl., 215 (1997), pp. 415-422.

[23] R. C. SMITH, G. A. Bogar, K. L. Bowers, AND J. Lund, The sinc-Galerkin method for fourth-order differential equations, SIAM J. Numer. Anal., 28 (1991), pp. 760-788. 
ETNA

Kent State University and

Johann Radon Institute (RICAM)

[24] A. J. Sommese And C. W. Wampler, The Numerical Solution of Systems of Polynomials Arising in Engineering and Science, World Scientific, Hackensack, 2005.

[25] V. Thомée, Galerkin Finite Element Methods for Parabolic Problems, Springer, Berlin, 1997.

[26] - Finite difference methods for computing eigenvalues of fourth order boundary value problems, Internat. J. Math. Math. Sci., 9 (1986), pp. 137-143.

[27] J. VERSCHELDE, Algorithm 795: Phcpack: A general-purpose solver for polynomial systems by homotopy continuation, ACM Trans. Math. Software, 25 (1999), pp. 251-276.

[28] A.-M. WAZWAZ, The numerical solution of special fourth-order boundary value problems by the modified decomposition method, Int. J. Comput. Math., 79 (2002), pp. 345-356.

[29] Y. S. YANG, Fourth-order two-point boundary value problems, Proc. Amer. Math. Soc., 104 (1988), pp. 175180.

[30] X. ZHANG, B. YU, AND J. ZHANG, Proof of a conjecture on a discretized elliptic equation with cubic nonlinearity, Sci. China Math., 56 (2013), pp. 1279-1286.

[31] X. ZHANG, J. ZHANG, AND B. YU, Eigenfunction expansion method for multiple solutions of semilinear elliptic equations with polynomial nonlinearity, SIAM J. Numer. Anal., 51 (2013), pp. 2680-2699. 